\title{
Number of involved anatomic areas as a risk predictor in pediatric Hodgkin's lymphoma: a retrospective study
}

\author{
Adriana Morais, ${ }^{1}$ Mário Henrique M. Barros, ${ }^{2}$ Rocio Hassan, ${ }^{3}$ \\ Vera L. L. Morais, ${ }^{1}$ Maria Tereza C. Muniz ${ }^{4}$
}

\begin{abstract}
Objective: To determine if the number of involved anatomic areas can modify the standard risk groups in pediatric Hodgkin's lymphoma, identifying children who would benefit from a reduction in treatment intensity.

Methods: Retrospective study evaluating age, sex, histology, Ann-Arbor stage, presence of B symptoms, number of involved anatomic areas, risk grouping (favorable vs. unfavorable), and laboratory exams. All patients received doxorubicin-containing chemotherapy. Patients in complete remission for 5 years or longer were evaluated as for late effects.

Results: Sixty-nine patients (2-18 years) were included, $68 \%$ belonged to the unfavorable risk group. Overall survival and event-free survival were 94 and $87 \%$, respectively. Late effects were screened in 46 cases. Advanced stage and $\geq$ four involved anatomic areas had negative impact on event-free survival, while only the number of involved anatomic areas retained statistical significance when using Cox analysis (hazard ratio $=6.4,95 \% \mathrm{CI}=$ $1.08-38.33 ; p=0.04)$. Risk groups were adjusted by number of involved anatomic areas $(<$ four $/ \geq$ four involved anatomic areas), with a significant reallocation of patients $(p=0.008)$. Of the 30 patients with late effects, 21 were in the original unfavorable risk group and $14(66.6 \%)$ could have been reallocated to the favorable risk group based on the number of involved anatomic areas.

Conclusion: If re-stratification had been applied, a considerable number of children would have received less intensive treatment and, consequently, could have had lower chances of late effects. A prospective study could define if adjustment of risk group by number of involved anatomic areas would have any impact on survival rates.

J Pediatr (Rio J). 2009;85(3):236-242: Hodgkin's lymphoma, childhood, risk groups, involved anatomic areas, late effects.
\end{abstract}

\section{Introduction}

Hodgkin's lymphoma $(\mathrm{HL})$ is a disease typically affecting children and young adults, with more than $80 \%$ of patients being cured. ${ }^{1}$ The other side of this high cure rate is that a fraction of patients will receive excessive antineoplastic radiochemotherapy resulting in the well-recognized late effects of $\mathrm{HL}$ treatment. ${ }^{2-6}$ Current clinical and radiological characteristics used for risk stratification in most treatment centers lead to mistaken stratification in almost one third of patients. ${ }^{7}$ Prognostic factors in $\mathrm{HL}$ are, mostly, crude direct measures of tumor burden and activity (stage, number of involved lymph nodes, bulky disease, B symptoms) or indirect surrogate measures of tumor burden and activity based on laboratory parameters (hemoglobin, s-albumin levels) ${ }^{8,9}$ Clinical characteristics at presentation, ${ }^{10,11}$ as

1. MD. Oncohematology Pediatric Center, Hospital Universitário Oswaldo Cruz (HUOC), Recife, PE, Brazil.

2. MD. Bone Marrow Transplantation Center (CEMO), Instituto Nacional do Câncer (INCA), Rio de Janeiro, RJ, Brazil.

3. PhD. CEMO, INCA, Rio de Janeiro, RJ, Brazil.

4. Oncohematology Pediatric Center, HUOC, Recife, PE, Brazil. Instituto de Ciências Biológicas, Universidade de Pernambuco (UPE), Recife, Brazil.

Financial support: Swissbridge Foundation (Switzerland) and Coordenação de Aperfeiçoamento de Pessoal de Nível Superior (CAPES, Brazil).

No conflicts of interest declared concerning the publication of this article.

Suggested citation: Morais A, Barros MH, Hassan R, Morais VL, Muniz MT. Number of involved anatomic areas as a risk predictor in pediatric Hodgkin's lymphoma: a retrospective study. J Pediatr (Rio J). 2009;85(3):236-242.

Manuscript received Dec 29 2008, accepted for publication Mar 042009.

doi:10.2223/JPED.1891 
well as protein immunoexpression ${ }^{10,12-14}$ and Epstein-Barr virus (EBV) association, ${ }^{15-17}$ have also been identified as prognostic factors in several studies.

However, when sufficiently intensive treatment for advanced stages is employed, adverse prognostic factors tend to disappear. ${ }^{9}$ Thus, the identification of clinical and biological factors that allow discrimination of patients who may undergo a reduction in treatment intensity is a current goal to reduce late effects in $\mathrm{HL}$.

The objective of this single-center study was to identify pretreatment characteristics that could be useful for adjustment of risk stratification in children with $\mathrm{HL}$.

\section{Materials and methods \\ Patients}

Sixty-nine patients up to 18 years old diagnosed with $\mathrm{HL}$ in the Pediatric Oncology Center (Centro de Oncohematologia Pediátrico, CEONHPE) of Oswaldo Cruz University Hospital, Recife, state of Pernambuco, Brazil, between March 1980 and January 2006 were included in this study. Inclusion was based on the availability of formalin-fixed paraffin-embedded $\mathrm{HL}$ tissue and complete clinical data for retrospective analysis. This study was approved by the Institutional Ethics Board.

\section{Histopathology}

HL diagnosis was reviewed according to the morphological criteria of the World Health Organization classification ${ }^{18}$ and confirmed by immunohistochemical staining, using CD30 (clone Ki-1, dilution, 1:40), CD15 (clone C3D-1, 1:50) and CD20 (clone L-26, 1:1000, Dako, Glostrup, Denmark).

\section{Staging and laboratory evaluation}

All patients were assessed before starting therapy by means of clinical history, complete physical examination, blood counts and biochemical profile, chest $x$-ray films, computed tomography of chest and abdomen and unilateral bone marrow biopsy. Staging followed the Ann-Arbor classification system. ${ }^{19} \mathrm{~B}$ symptoms were characterized by fever $\geq 38^{\circ} \mathrm{C}$ for at least 3 consecutive days, night sweats and loss of weight. Disease risk was classified as favorable (I, IIA, IIIA) or unfavorable (IIB, IIIB, IV). Anemia was defined as hemoglobin $<13 \mathrm{~g} / \mathrm{dL}$ ( $<11.5 \mathrm{~g} / \mathrm{dL}$ for female patients in reproductive age). Lactate dehydrogenase ( $L D H)$ and erythrocyte sedimentation rate (ESR) were considered high when higher than $500 \mathrm{IU} / \mathrm{L}$ and $40 \mathrm{~mm} / \mathrm{h}$, respectively.

\section{Treatment}

All patients received doxorubicin-containing chemotherapy. Alternating AdriamycinTM, bleomycin, vinblastine and dacarbazine/vincristine, procarbazine, prednisone and Adriamycin ${ }^{T M}$ (ABVD/OPPA) were administered to 33 children (47.8\%) until 1996 and ABVD alone, to 36 children (52.2\%) after 1996, respectively. ${ }^{20,21}$ Children in the favorable risk group were treated with three cycles of chemotherapy, while those in the unfavorable risk group received six cycles. Low-dose involved-field radiotherapy was administered to all patients, except for one patient with stage IA, at the end of chemotherapy protocol (15 Gy for patients that achieved complete response and $25 \mathrm{~Gy}$ for those with bulky disease at presentation or local residual disease at the end of chemotherapy).

\section{Variables analyzed as potential prognostic factors}

Pretreatment variables were: age, sex, histology, Ann-Arbor stage, presence of $B$ symptoms, number of involved anatomic areas (IAA), risk grouping (favorable vs. unfavorable), LDH level, ESR and anemia. Albumin level, white blood cell counts and biochemical profile were unavailable for more than $10 \%$ of the patients; therefore, these data were not analyzed as prognostic factors.

The following sites were taken into account for the calculation of the number of IAA: Waldeyer's ring, cervical and/or supraclavicular lymph nodes (right or left, separately), axillary and/or infraclavicular lymph nodes (right or left separately), epitrochlear nodes (right or left, separately), mediastinal plus hilar lymph nodes (one site), para-aortic and/or retrocrural lymph nodes (one site), porta hepatis lymph nodes (one site), mesenteric (one site), iliac and/or inguinal/femoral lymph nodes (right or left, separately), spleen and splenic hilar lymph nodes (one site). Each extranodal site was considered separately. Each lung was also considered as a separate site. In the case of multiple bone lesions, bone was considered as one involved site.

\section{Late effect evaluation}

Patients in complete remission for 5 years or more were recalled by the Cured Evaluation Program at the CEONHPE. Late effect screening consisted of hematological and hormone analyses, fertility tests, cardiac evaluation by annual electrocardiogram and echocardiogram until the end of growth, pulmonary function tests, audiometry, renal function tests and psychological evaluation, as described. ${ }^{3}$

\section{Statistical analysis}

Associations between categorical variables were tested using two-tailed Pearson's chi-square and Fisher's exact tests. Significance was set at $p<0.05$. Event-free survival (EFS) was estimated as the interval (in months) from diagnosis to relapse or death by any cause or, alternatively, to the last follow-up. Overall survival (OS) refers to the lapse (in months) from diagnosis to death or last follow-up. Survival distributions were estimated according to the Kaplan-Meier method, 22 and differences were assessed by the log-rank test. Multivariate analysis 
using Cox proportional hazard regression ${ }^{23}$ was performed to determine the independent prognostic factors influencing EFS. Data were analyzed with SPSS v.13.0.

\section{Results \\ Clinical and demographic characteristics at presentation}

Of the 69 patients included in this study, 49 (71\%) were male ( $M: F 2.45: 1$ ). Ages ranged from 2 to 18 years (median 11 years), with $56.5 \%$ (39/69) of patients in the $>10$ years group. Most of the patients had disease stage I or II $(36 / 69,52.1 \%)$. B symptoms were observed in $47 / 69$ $(68.1 \%)$ cases. The analysis by risk group showed that $22 / 69$ patients were $(31.9 \%)$ in the favorable and $47 / 69$ $(68.1 \%)$ in the unfavorable risk group. Nodular sclerosis was the most common histological subtype (51/69 cases, $74 \%$ ), followed by mixed cellularity (17/69, 24.6\%). Hemoglobin levels were evaluated in 59 children, varying from 7.4 to $16 \mathrm{~g} / \mathrm{dL}$ (median 11.3). Anemia was observed in 23 patients (33.3\%) (Table 1$)$.

The number of IAA varied from one to eight (median two). When the group was classified according to the number of IAA ( $<$ four or $\geq$ four IAA), 17 children $(24.6 \%$ ) showed $\geq$ four IAA, while $52(75.4 \%)$ showed $<$ four IAA. A number of $\geq$ four IAA showed association with stages III/IV (16/17, $94 \%, p=0.0001)$, B symptoms $(16 / 17,94 \%, p=0.008)$, unfavorable risk group $(16 / 17,94 \%, p=0.0001)$, and anemia $(10 / 15,66.6 \%, p=0.01)$. In multivariate analysis, the number of IAA was associated only with stage ( $95 \% \mathrm{CI}$ -0.5 to $-0.1, p=0.0005)$.

\section{Treatment response and survival analysis}

Sixty patients $(87 \%)$ finished the treatment and did not relapse, while six patients $(8.7 \%)$ relapsed after the end of treatment and three (4.3\%) during the first-line treatment. Sixty-five patients are alive and in complete response $(94.2 \%)$, three $(4.3 \%)$ died because of $\mathrm{HL}$ progression and one died of a chondrosarcoma secondary to $\mathrm{HL}$ treatment. The median length of follow-up was 82 months (12-332 months); the shortest follow-up (12 m) involved a patient who died of disease progression. The OS and EFS rates for the whole group were 94.2 and $87 \%$, respectively (Figure 1 ).

Since both protocols used in the treatment of the patients (ABVD/OPPA and ABVD) are considered equivalent in their ability to induce complete response $\mathrm{e}^{20,21}$ and no differences either in survival $(p=0.6)$ or in demographic and clinical characteristics (data not shown) were observed between the two groups, all patients were included in the survival analyses. Children with stage IV showed the worst EFS when compared to children with other stages (40 vs. 100, 89.7 and $89.3 \%$ for stages I, II and III, respectively; $p=0.002)$. The number of IAA showed a strong effect on EFS; $64.7 \%$ in children with $\geq$ four IAA compared with $94.2 \%$ in patients with < four IAA ( $p=0.0001)$; while anemia showed a borderline effect (78.3 vs. $94.4 \%$ in children without anemia; $p=0.06)$. Sex, age group, risk group, $B$ symptoms, LDH levels, ESR and histological subtypes had no significant effects on EFS (Table 1$)$. Stage IV (60\%, $p=0.001$ ), anemia ( 87 vs. $100 \%$ in nonanemic patients; $p=0.03$ ) and $\geq$ four IAA (82.4 vs. $98.1 \%$ in < four IAA; $\mathrm{p}=0.001$ ) significantly impacted on OS.

A Cox analysis was performed including the significant EFS variables in univariate analyses. The number of IAA was an independent prognostic factor (hazard ratio [HR] 6.4, 95\%CI 1.08-38.33; $p=0.04$ ), while stage lost significance.

The former results suggested that the number of IAA could be used to improve the risk stratification system. Thus, the original risk groups were adjusted by the number of IAA: cases with unfavorable disease and $<$ four IAA were reclassified as "favorable disease" (except for extranodal disease that was maintained as unfavorable), and children with favorable disease and $\geq$ four IAA were reclassified to the unfavorable disease group. With this reallocation, only one of the 22 patients initially in the favorable risk group was reallocated to the adjusted unfavorable risk group; however, of the 47 patients originally in the unfavorable risk group, 31 (66\%) were reallocated to the favorable risk group, while $16(34 \%)$ remained in the unfavorable risk group ( $p=0.008$ ) (Figure 2 ). The adjusted risk groups were better predictors of EFS than the original ones: 94.2 vs. $64.7 \%$ of EFS for the adjusted favorable and unfavorable risk groups, respectively ( $p=0.0005)$, compared with 95.5 vs. $83 \%$ of EFS ( $p=0.1$ ) for the original risk groups.

To avoid statistical bias due to the inclusion of patients submitted to different intensity treatments, EFS was analyzed separately for each risk group. No differences were observed among the patients who received low-intensity treatment: the only relapsed patient had $<$ four IAA and was originally in the favorable risk group $(p=0.4)$. Conversely, in the group of intensively treated patients, results were comparable to those observed in the whole group ( 93.5 vs. $62.5 \%$ of EFS, adjusted favorable and unfavorable risk groups, respectively; $p=0.003$ ). A Cox regression was performed in the intensive treatment group, reinforcing the independent prognostic impact of the adjusted risk group for unfavorable disease (HR 6.7, 95\%CI 1.07-42.1; $\mathrm{p}=0.04$ ).

Forty-six patients responded to recall for late effect screening. Thirty patients (65\%) exhibited some kind of late effects, cardiotoxicity and endocrine dysfunction being the most frequent. Four children developed a second neoplasm (chondrosarcoma, thyroid carcinoma, rhabdomyosarcoma and myelodysplastic syndrome, respectively) (Table 2). Most patients with late effects were in the original unfavorable risk group (21/30, $70 \%)$. Fourteen of the 31 children (45\%) 
Table 1 - Clinical and histological characteristics of Hodgkin's lymphoma patients

\begin{tabular}{|c|c|c|c|}
\hline Variable & $\begin{array}{c}\text { Cases analyzed } \\
\text { n ( } \%)\end{array}$ & $\begin{array}{c}\text { EFS } \\
\% \text { (no. events/total) }\end{array}$ & $\begin{array}{c}\text { Univariate } \\
\text { analysis }\end{array}$ \\
\hline \multicolumn{4}{|l|}{ Age (years) } \\
\hline Median (range) & $11(2-18)$ & & $p=0.2$ \\
\hline$\leq 10$ & $30 / 69(43.5)$ & $80(6 / 30)$ & \\
\hline$>10$ & $39 / 69$ (56.5) & $92.3(3 / 39)$ & \\
\hline Sex & & & $p=0.1$ \\
\hline Male & 49/69 (71) & $89.8(5 / 49)$ & \\
\hline Female & 20/69 (29) & $80(4 / 20)$ & \\
\hline Anemia & & & $p=0.06$ \\
\hline Yes & 23/59 (39) & $78.3(5 / 23)$ & \\
\hline No & $36 / 59(61)$ & $94.4(2 / 36)$ & \\
\hline \multicolumn{4}{|l|}{$\mathrm{LDH}$} \\
\hline Median & & & $p=0.2$ \\
\hline Normal & $30 / 59(50.8)$ & $93.3(2 / 30)$ & \\
\hline High & $29 / 50(49.2)$ & $86.2(4 / 29)$ & \\
\hline \multicolumn{4}{|l|}{ ESR } \\
\hline Median (range) & $59.5(2-140)$ & & $p=0.2$ \\
\hline Normal & $18 / 59(30.5)$ & $100(0)$ & \\
\hline High & $41 / 59(69.5)$ & $87.8(5 / 41)$ & \\
\hline \multicolumn{4}{|l|}{ Number of anatomic areas } \\
\hline Median (range) & $2(1-8)$ & & $p=0.0005$ \\
\hline$<4$ & $52 / 69(75.4)$ & $94.2(3 / 52)$ & \\
\hline$\geq 4$ & $17 / 69(24.6)$ & $64.7(6 / 17)$ & \\
\hline Stage & & & $p=0.002$ \\
\hline $\mathrm{I}$ & $7 / 69(10.1)$ & $100(0)$ & \\
\hline II & $29 / 69(42)$ & $89.7(3 / 29)$ & \\
\hline III & $28 / 69(40.6)$ & $89.3(3 / 28)$ & \\
\hline IV & $5 / 69(7.2)$ & $40(2 / 5)$ & \\
\hline B symptoms & & & $p=0.1$ \\
\hline Yes & 22/69 (31.9) & $83(8 / 47)$ & \\
\hline No & $47 / 69(68.1)$ & $95.5(1 / 22)$ & \\
\hline Risk group & & & $p=0.1$ \\
\hline Favorable & 22/69 (31.9) & $95.5(1 / 22)$ & \\
\hline Unfavorable & $47 / 69(68.1)$ & $83(8 / 47)$ & \\
\hline Histopathological diagnosis & & & $p=0.8$ \\
\hline Nodular sclerosis & $51 / 69(73.9)$ & $88.2(6 / 51)$ & \\
\hline Mixed cellularity & $17 / 69(24.6)$ & $82.4(3 / 17)$ & \\
\hline Lymphocyte-depleted & $1 / 69(1.4)$ & $100(0)$ & \\
\hline
\end{tabular}

$\mathrm{EFS}=$ event-free survival; $\mathrm{ESR}=$ erythrocyte sedimentation rate; $\mathrm{LDH}=$ lactate dehydrogenase

B symptoms $=$ fever $\geq 38^{\circ} \mathrm{C}$ for at least 3 consecutive days, night sweats and loss of weight.

reallocated from the original unfavorable risk group to the favorable risk group based on the number of IAA exhibited late effects $(p=0.005)$

\section{Discussion}

In respect to clinical outcome, our results with a risk-adapted, homogeneously treated group of patients achieved survival rates comparable to those obtained in developed countries $2,5,24,25$ and in the most developed region of Brazil. ${ }^{21}$ The late effects were also similar to those described in other studies that treated children with the same protocols. ${ }^{1-6}$ With the current risk-stratified treatment for $\mathrm{HL}$, long-term disease-free survivals (DFS) were 85 to $100 \%$ in patients with early-stage disease, and more than $60 \%$ in those with advanced disease. ${ }^{26}$ The German Hodgkin Study Group showed an improvement of survival for patients with advanced stages when more intensive treatments were applied. ${ }^{4}$ However, it is well known that higher survival rates are accompanied by late effects of treatment, mostly in patients treated during childhood. ${ }^{5}$ For example, the excellent results reached in high stages by the German Hodgkin Study Group were associated with a higher frequency of secondary acute myeloid leukemia/myelodysplastic syndrome. ${ }^{4}$ Thus, it is accepted that the challenge today in $\mathrm{HL}$ treatment is to minimize late toxicity without compromising the excellent survival rates. 

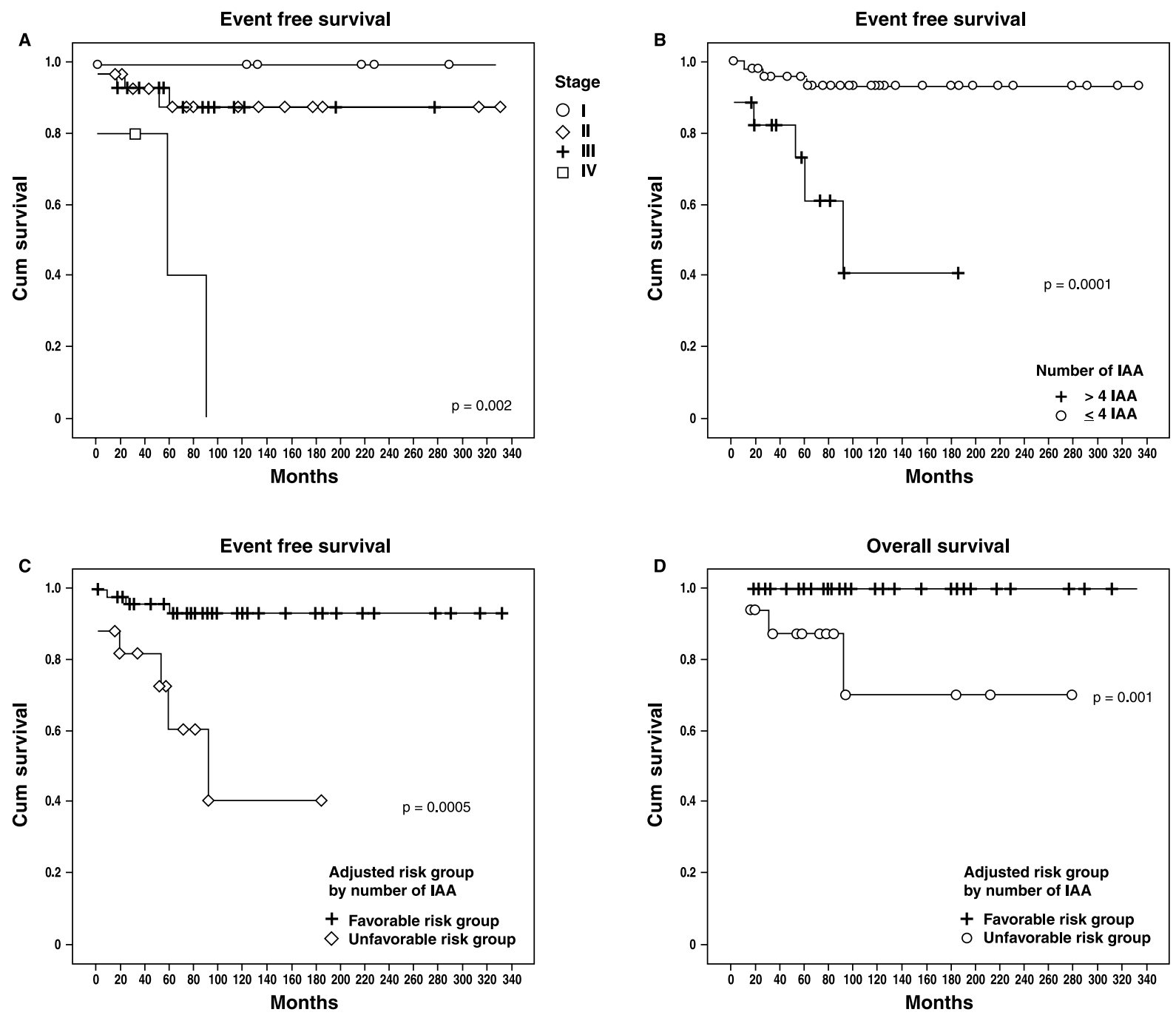

Cum = cumulative; $I A A=$ involved anatomic areas.

Figure 1 - Survival analysis

Table 2 - Distribution of patients with late effects by risk group

\begin{tabular}{|c|c|c|c|}
\hline \multirow[b]{2}{*}{ Late effects } & \multirow[b]{2}{*}{ Affected/evaluated patients $(\%)$} & \multicolumn{2}{|c|}{ Risk group* } \\
\hline & & Favorable (\%) & Unfavorable (\%) \\
\hline Cardiotoxicity & $18 / 37(48.6)$ & $4 / 18(22.2)$ & $14 / 18(77.8)$ \\
\hline Endocrine alterations & $9 / 40(22.5)$ & $3 / 9(33.4)$ & $6 / 9(66.6)$ \\
\hline Bone alterations & $1 / 40(2.5)$ & $1 / 1(100)$ & 0 \\
\hline Pulmonary toxicity & $2 / 40(5)$ & $1 / 2(50)$ & $1 / 2(50)$ \\
\hline Renal toxicity & $2 / 40(5)$ & $1 / 2(50)$ & $1 / 2(50)$ \\
\hline Audiologic alterations & $2 / 40(5)$ & 0 & $2 / 2(100)$ \\
\hline Cosmetic alterations $^{\dagger}$ & $6 / 44(13.6)$ & $2 / 6(33.3)$ & $4 / 6(66.7)$ \\
\hline Second neoplasm & $4 / 44(9.1)$ & $2 / 2(50)$ & $2 / 2(50)$ \\
\hline Total & $30 / 46(65.2)$ & $9 / 30(30)$ & $21 / 30(70)$ \\
\hline
\end{tabular}

* Original risk group (without number of involved anatomic sites reallocation).

$\dagger$ Atrophy of skin secondary to radiotherapy. 


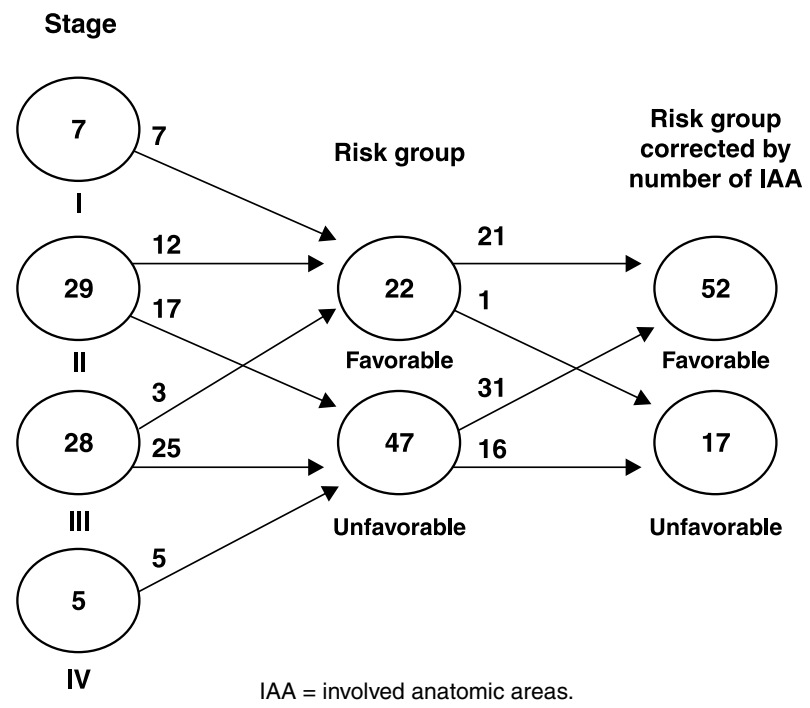

Figure 2 - Adjusted risk groups by number of IAA

It was showed that children with more than four IAA had a 6.4-fold increased risk of unfavorable outcome. The results agree with those of Vassilakopoulos et al., who showed a significant influence of this variable in DFS prediction in adolescents and adults with advanced-stage disease. ${ }^{10}$ Conversely, a previous study on childhood $\mathrm{HL}$ did not show a significant effect of IAA on DFS. 27 The discordance between the results of this current study and those reported by Oguz et al. ${ }^{27}$ might be due to the fact that the latter study only considered the number of involved nodal sites, suggesting that the number of total involved sites (nodal and extranodal) may be a better predictor of prognosis in pediatric HL.

The number of IAA may be a surrogate indicator of tumor burden, a strong adverse prognostic factor for $\mathrm{HL}$ albeit a difficult variable to quantify or even estimate. ${ }^{27-31}$ Staging is unable to predict tumor burden with more accuracy because it does not consider the number of affected anatomic sites. For example, a stage IIA patient with cervical and axillary nodes, both on the same body side, would have a lower tumor burden than another patient with no symptoms and cervical and axillary bilateral involvement, who would also be staged as IIA. Therefore, it is possible that some children with advanced-stage disease are overtreated. ${ }^{9}$ The results of this study indicate that a subgroup of patients with better prognosis can be identified among the unfavorable-risk patients, based on tumor burden as defined by number of IAA, and suggest that the main negative consequence of tailoring chemotherapy based only on the stage and/or risk group (stage combined with presence of B symptoms) is the overtreatment of a group of patients stratified as advanced-disease, without having a high tumor burden.

The limitation of this retrospective study is the absence of early response evaluation by positron emission tomography, a current conduct of the modern HL therapy, but not feasible to all oncology centers from developing countries. Because of this, studies that try to obtain the best methodology to estimate the tumor burden and define therapeutic conducts are still important.

The present study aimed, through a balance of risk between treatment response and late effects in a carefully followed cohort, to identify pretreatment factors for risk adjustment in unfavorable disease. The number of IAA is an easily obtainable clinical parameter that could be potentially incorporated to stratify patients with the aim of reducing late toxicity in pediatric $\mathrm{HL}$. If re-stratification had been applied in this study group, a considerable number of children would have received less intensive treatment and, consequently, could have had lower chances of late effects. Undoubtedly, a prospective study is mandatory to confirm if the proposed reallocation will modify the survival rates.

\section{Acknowledgements}

This study was partially supported by the Swissbridge Foundation, Switzerland, and by Coordenação de Aperfeiçoamento de Pessoal de Nível Superior (CAPES), Brazil. 


\section{References}

1. Hodgson DC. Hodgkin Lymphoma: the follow-up of long-term survivors. Hematol Oncol Clin North Am. 2008;22:233-44.

2. Thomson $A B$, Wallace WH. Treatment of paediatric Hodgkin's disease. A balance of risks. Eur J Cancer. 2002;38:468-77.

3. Barros MH, Morais VL, Soares SM, Leite EP, Ramos SV. Late effects in Brazil: experience of the Cured Evaluation Program. Med Pediatr Oncol. 2003;41:361 (Abstract)

4. Diehl V, Klimm B, Re D. Hodgkin lymphoma: a curable disease: what comes next? Eur J Haematol Suppl. 2005;(66):6-13.

5. Schwartz CL. Special issues in pediatric Hodgkin's disease. Eur J Haematol Suppl. 2005;(66):55-62.

6. Hodgson DC, Hudson MM, Constine LS. Pediatric hodgkin lymphoma: maximizing efficacy and minimizing toxicity. Semin Radiat Oncol. 2007;17:230-42.

7. Montalbán C, García JF, Abraira V, González-Camacho L, Morente $\mathrm{MM}$, Bello JL, et al. Influence of biologic markers on the outcome of Hodgkin's lymphoma: a study by the Spanish Hodgkin 's Lymphoma Study Group. J Clin Oncol. 2004;22:1664-73.

8. Specht L, Nordentoft AM, Cold S, Clausen NT, Nissen NI. Tumor burden as the most important prognostic factor in early stage Hodgkin's disease. Relations to other prognostic factors and implications for choice of treatment. Cancer. 1988;61:1719-27.

9. Hasenclever, D. The disappearance of prognostic factors in Hodgkin's disease. Ann Oncol. 2002;13 Suppl 1:75-8.

10. Vassilakopoulos TP, Angelopoulou MK, Siakantaris MP, Kontopidou FN, Dimopoulou MN, Barbounis $A$, et al. Prognostic factors in advanced stage Hodgkin's lymphoma: the significance of the number of involved anatomic sites. Eur J Haematol. 2001;67:279-88

11. Zander T, Wiedenmann S, Wolf J. Prognostic factors in Hodgkin 's Iymphoma. Ann Oncol. 2002;13 Suppl 1:67-74

12. Morente MM, Piris MA, Abraira V, Acevedo A, Aguilera B, Bellas C, et al. Adverse clinical outcome in Hodgkin 's disease is associated with loss of retinoblastoma protein expression, high Ki67 proliferation index, and absence of Epstein-Barr virus-latent membrane protein 1 expression. Blood. 1997;90:2429-36.

13. Rassidakis GZ, Medeiros LJ, Viviani S, Bonfante V, Nadali GP, Vassilakopoulos TP, et al. CD20 expression in Hodgkin and Reed-Sternberg cells of classical Hodgkin 's disease: associations with presenting features and clinical outcome. J Clin Oncol. 2002;20:1278-87.

14. Sánchez-Aguilera $A$, Montalbán $C$, de la Cueva $P$, Sánchez-Verde L, Morente MM,García-Cosío M, et al. Tumor microenvironment and mitotic checkpoint are key factors in the outcome of classic Hodgkin lymphoma. Blood. 2006;108:662-8.

15. Engel M, Essop MF, Close P, Hartley P, Pallesen G, Sinclair-Smith C. Improved prognosis of Epstein-Barr virus associated childhood Hodgkin's Iymphoma: study of 47 South African cases. J Clin Pathol. 2000;53:182-6.

16. Claviez A, Tiemann M, Lüders $H$, Krams M, Parwaresch $R$, Schellong $\mathrm{G}$, et al. Impact of latent Epstein-Barr virus infection on outcome in children and adolescents with Hodgkin's Iymphoma. J Clin Oncol. 2005;23:4048-56.

17. Jarrett RF, Stark GL, White J, Angus B, Alexander FE, Krajewski AS, et al. Impact of tumor Epstein-Barr virus status on presenting features and outcome in age-defined subgroups of patients with classic Hodgkin lymphoma: a population-based study. Blood. 2005; 106:2444-51.
18. Jaffe ES, Harris NL, Stein $\mathrm{H}$, et Vardiman JW, eds. World Health Organization Classification of Tumors. Pathology and Genetics of Tumours of Hematopoietic and Lymphoid Tissues. Lyon: IARC Press; 2001.

19. Carbone PP, Kaplan HS, Musshoff K, Smithers DW, Tubiana M. Report of the Committee on Hodgkin's Disease Staging Classification. Cancer Res. 1971;31:1860-1.

20. Hudson M, Onciu M, Donaldson S. Hodgkin lymphoma. In: Pizzo P, Poplack D, editors. Principles and practice of pediatric oncology. 5th ed. Philadelphia, PA: Lippincott Williams \& Wilkins, 2006. p.: 695-721.

21. Viani GA, Castilho MS, Novaes PE, Antonelli CG, Ferrigno R, Pellizzon $\mathrm{CA}$, et al. Chemotherapy followed by low dose radiotherapy in childhood Hodgkin's disease: retrospective analysis of results and prognostic factors. Radiat Oncol. 2006;1:38.

22. Cox, DR. Regression Models and life tables (with discussion). J R Stat Soc B. 1972;34:187-220.

23. Kaplan E, Meier P. Non Parametric estimation from incomplete observations. J Am Stat Assoc. 1958;53:457-81.

24. Oberlin $O$, Leverger $G$, Pacquement $H$, Raquin MA, Chompret A, Habrand $\mathrm{J}$, et al. Low-dose radiation therapy and reduced chemotherapy in childhood Hodgkin's disease: the experience of the French Society of Pediatric Oncology. J Clin Oncol. 1992;10:1602-8.

25. Schellong G, Pötter R, Brämswig J, Wagner W, Prott FJ, Dörffel W, et al. High cure rates and reduced long-term toxicity in pediatric Hodgkin's disease: the German-Austrian multicenter trial DALHD-90. The German-Austrian Pediatric Hodgkin's Disease Study Group. J Clin Oncol. 1999;17:3736-44

26. Smith RS, Chen $\mathrm{Q}$, Hudson MM, Link MP, Kun L, Weinstein $\mathrm{H}$, et al. Prognostic factors for children with Hodgkin's disease treated with combined-modality therapy. J Clin Oncol. 2003;21:2026-33.

27. Oguz A, Karadeniz C, Okur FV, Citak EC, Pinarli FG, Bora H, et al. Prognostic factors and treatment outcome in childhood Hodgkin disease. Pediatr Blood Cancer. 2005;45:670-5.

28. Gobbi PG, Ghirardelli ML, Solcia M, Di Giulio G, Merli F, Tavecchia $L$, et al. Image-aided estimate of tumour burden in Hodgkin's disease: evidence of its primary prognostic importance. J Clin Oncol. 2001; 19:1388-94.

29. Specht L, Nissen NI. Prognostic factors in Hodgkin's disease stage IV. Eur J Haematol. 1988;41:359-67.

30. Specht L, Nissen NI. Prognostic factors in Hodgkin's disease stage III with special reference to tumour burden. Eur J Haematol. 1988;41:80-7.

31. Specht L, Nissen NI. Hodgkin's disease and age. Eur J Haematol. $1989 ; 43 ; 127-35$.

Correspondence:

Maria Tereza Cartaxo Muniz

Laboratório de Biologia Molecular

Centro de Oncohematologia Pediátrica

Hospital Universitário Oswaldo Cruz

Rua Arnóbio Marques, 310, Santo Amaro

CEP 50100-130 - Recife, PE - Brazil

E-mail: tcartaxo.upe@hotmail.com 